Result(s)* Results show no statistical significance in difference between age of patients in the moment of surgical treatment $(p=0.126)$. There was statistical significant difference in positive peritoneal cytology between group with endometrioid compared with nonendometrioid endometrial carcinoma $(p=0.000)$ group. Also there was, statistical significant difference between group with endometrial cancer confined to uterus compare with patients with carcinoma beyond the uterus $(p=0.000)$ and statistically significant was confirmed with high tumor grade $(p=0.000)$.

Conclusion* The positive peritonal cytology was found statisticaly significance in the group of the patients with non endometroides type of the tumor, highest tumor grade and higher FIGO classification. For these group of patients have to consider to still recommend using peritoneal washings cytology analyses during surgery.

\section{EPIGENETIC METHYLATION IN ENDOMETRIAL CANCER (EC): AN UMBRELLA REVIEW OF PUBLISHED EVIDENCE}

${ }^{1} \mathrm{~K}$ Rallis*, ${ }^{2} \mathrm{M}$ Sideris, ${ }^{1} \mathrm{Y} A$ Wang, ${ }^{1} \mathrm{C}$ Hillyar, ${ }^{1} \mathrm{~S}$ Makker, ${ }^{3} \mathrm{E}$ Emin, ${ }^{2} \mathrm{~T}$ Mould. ${ }^{1}$ Barts and the London School of Medicine and Surgery, London, UK; ${ }^{2}$ University College London Hospital, London, UK; ${ }^{3}$ Kingston University, Kingston, UK

\subsection{6/ijgc-2021-ESG0.221}

Introduction/Background* EC is the commonest gynaecological malignancy in developed countries accounting for 97,000 deaths annually worldwide. Methylation of DNA, histones, and micro-RNA (miRNA) are important epigenetic events which predominantly repress gene expression, reversibly, thus altering the tumour-cellular phenotype. We performed an umbrella review of relevant narrative (NR) and systematic reviews (SR) to identify prognostic and/or predictive methylation biomarkers for clinical decision-making.

Methodology We followed the PRISMA guidelines and prospectively registered our protocol with PROSPERO (CRD42021225841). We searched MEDLINE from inception to December $4^{\text {th }}, 2020$, for NRs and SRs, published in English, discussing the association between methylation of DNA, histones or miRNA and early detection, prognosis or treatment response in EC. Three reviewers extracted and assessed the quality of the evidence for qualitative synthesis.

Result(s)* Our search yielded 86 publications. We included 19 articles: 2 SRs with meta-analysis and 17 NRs. Type I and II EC exhibit distinct methylation profiles of gene silencing. Type I EC contains near-diploid cells (vs. aneuploid in type II) and its progression is more strongly associated with epigenetic changes. Global hyper- or hypomethylation and CIMP are described. Gene promoter hypermethylation predominates especially in hormone receptor, MLH1, MGMT, CDKN2A/ P16, WNT/b-catenin/E-cadherin, PIK3CA/MAPK/RAS, and FGF pathway components. Ribosomal DNA hypomethylation, DNA aneuploidy, and lack of $\mathrm{CDH} 13$ hypermethylation predict poor prognosis. Dysregulated miRNA expression and processing differentially promotes oncogenesis. Histone methyltransferases (EZH2) and demethylases (LSD1, KDM4A/B) are overexpressed correlating with aggressiveness. Meta-analyses report significant associations with RASSF1A promoter methylation and $C D K N 2 A / p 16$ hypermethylation which occur early in carcinogenesis potentially aiding non-invasive detection.

Conclusion* As type I EC primarily affects younger women, some of whom have yet to complete their family, prognostication via (epigenetic) biomarkers is important for treatment stratification. Predicting response to conservative (progesterone) treatment via methylation biomarkers could help identify eligible women for 'watch and wait' until they complete their family. Artificial intelligence (AI) could facilitate such efforts.

\section{MOLECULAR SUBTYPE DIAGNOSIS OF ENDOMETRIAL CARCINOMA USING AN NGS PANEL}

${ }^{1 ; 2}$ J Huvila* ${ }^{1 ; 2} \mathrm{~K}$ Orte, ${ }^{1 ; 2} \mathrm{P}$ Vainio, ${ }^{3} \mathrm{~T}$ Mettälä, ${ }^{3} \mathrm{~T}$ Joutsiniemi, ${ }^{3} \mathrm{~S}$ Hietanen. ${ }^{1}$ Turku University Hospital, Department of Pathology, Finland; ${ }^{2}$ University of Turku, Department of Biomedicine, Finland; ${ }^{3}$ Turku University Hospital, Department of Obstetrics and Gynecology, Finland

\subsection{6/ijgc-2021-ESGO.222}

Introduction/Background* The molecular classification of endometrial carcinoma (EC) is integrated in the new ESMO-ESGOESTRO treatment guidelines and is needed for appropriate treatment planning. An NGS panel can be used for molecular classification, assessing POLE mutation status, microsatellite instability (MSI) and TP53 mutation status. We describe the process of molecular classification on $60 \mathrm{EC}$ cases based on
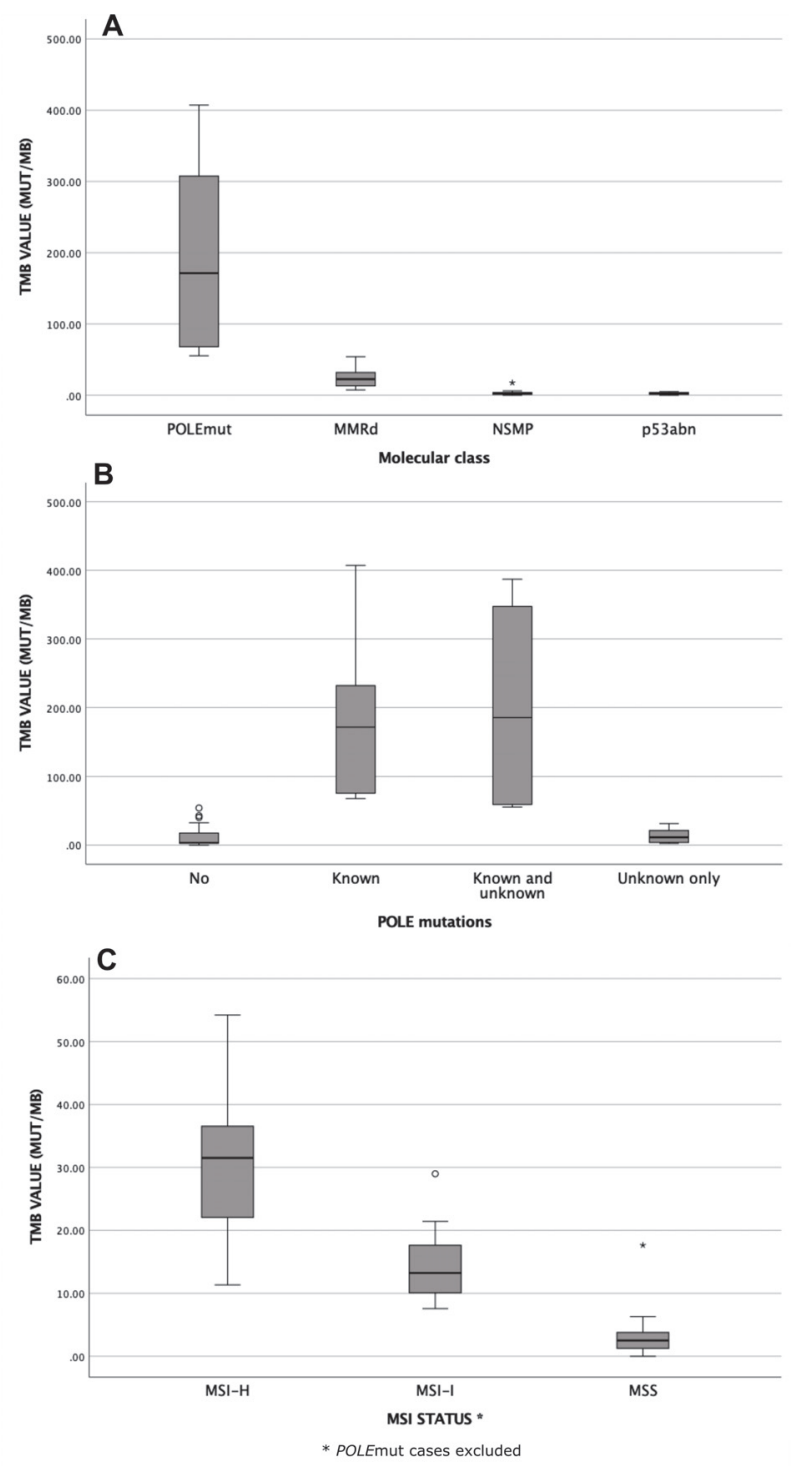

Abstract 1006 Figure 1 